DOI: https://doi.org/10.46296/yc.v5i9edespsoct.0112

\title{
COMPETENCIAS DEL DOCENTE CREATIVO PARA LA ENSEÑANZA DE UNA SEGUNDA LENGUA
}

\section{CREATIVE TEACHER COMPETENCIES FOR SECOND LANGUAGE TEACHING}

\author{
Véliz-Zambrano Alexandra del Rocío ${ }^{1}$; Game-Varas Cinthya Isabel ${ }^{2}$ \\ ${ }^{1}$ Universidad San Gregorio de Portoviejo USGP. Portoviejo Ecuador. Correo: \\ albodasije@gmail.com. ORCID ID: https://orcid.org/0000-0001-7567-1958 \\ 2 Universidad San Gregorio de Portoviejo USGP. Portoviejo Ecuador. Correo: \\ cinthya.gamev@gmail.com. ORCID ID: https://orcid.org/0000-0002-4877-1192
}

\section{Resumen}

El objetivo de la presente investigación se proyectó en identificar los retos y desafíos del rol del docente creativo en la enseñanza de una segunda lengua y determinar la importancia que tiene en el desarrollo de los aprendizajes mediante una educación a distancia, fundamentado en el conocimiento a través de la red, siendo el docente quien acompaña al estudiante en este proceso de aprendizaje. Se inicia desde el análisis de diversos autores en relación con estas concepciones que consolida y afirma su pertinencia con contenidos educativos y estrategias innovadoras en la docencia ofreciendo opciones de calidad a los estudiantes. Entre los tipos de investigación que se utilizaron, encontramos: No experimental, Descriptiva y Exploratoria, revisando bibliografías, artículos científicos y citas. Los métodos en que se apoyó esta investigación fueron; el Analítico - Deductivo. Dos cuestionarios sirvieron para la obtención de datos: la encuesta a los estudiantes y la entrevista a las autoridades y docentes del área de la segunda lengua. En su desenlace se refleja que el rol docente, la pedagogía, la didáctica y la creatividad, requieren espacios concretos para el análisis, reflexión y aplicación en todo el proceso de enseñanza aprendizaje; la enseñanza de una segunda lengua requiere de ese docente creativo, con identidad, con formación integral, con recursos y herramientas que permitan la práctica de todas las innovaciones inherentes a este proceso, y; la educación a distancia o virtual como una capacidad inherente al ser humano que requiere ser practicada para convertirla en habilidad.

Palabras claves: Competencias; creatividad; educación virtual; desempeño docente.

\begin{abstract}
The objective of this research was projected to identify the challenges and challenges of the role of the creative teacher in the teaching of a second language and to determine the importance it has in the development of learning through distance education, based on knowledge through of the network, being the teacher who accompanies the student in this learning process. It starts from the analysis of various authors in relation to these conceptions that consolidates and affirms their relevance with educational content and innovative strategies in teaching, offering quality options to students. Among the types of research that were used, we found: Non-experimental, Descriptive and Exploratory, reviewing bibliographies, scientific articles and citations. The methods on which this research was supported were; the Analytical - Deductive. Two questionnaires were used to obtain data: the student survey and the interview with the authorities and teachers in the second language area. In its outcome it is reflected that the teaching role, pedagogy, didactics and creativity require specific spaces for analysis, reflection and application throughout the teaching-learning process; The teaching of a second language requires that creative teacher, with identity, with comprehensive training, with resources and tools that allow the practice of all the innovations inherent to this process, and; distance or virtual education as an inherent capacity of the human being that needs to be practiced in order to turn it into a skill.
\end{abstract}

Keywords: Competences; creativity; virtual education; teaching performance.

Información del manuscrito:

Fecha de recepción: 22 de julio de 2021.

Fecha de aceptación: 24 de septiembre de 2021.

Fecha de publicación: 01 de octubre de 2021. 


\section{Introducción}

Tomando como punto de partida la educación en las instituciones educativas presentan transformaciones, desafíos, retos; el desarrollo imparable de tecnologías digitales y la democratización en el uso de Internet han sido uno de los cambios que más han transformado el contexto del proceso educativo. El conocimiento es una fuente de desarrollo para la preparación de los estudiantes, dentro de estos conocimientos se encuentran los actuales sistemas de información que han generado cambios en los perfiles de los actores de este proceso educativo, se precisan métodos que permitan enfocarse en las dimensiones de esta realidad que ayuden a que el sistema educativo se enfrente a los nuevos modelos. A nivel institucional surgen distintas tendencias que afectan directamente la función académica, por ello se busca la construcción de espacios que propicien el aprendizaje permanente y promuevan la generación, difusión y la creación de nuevos conocimientos, por lo que resulta imprescindible analizar sus repercusiones y ofrecer respuestas pertinentes a estos requerimientos.
De manera creciente la institución educativa fiscal Francisco Pacheco de la ciudad de Portoviejo, Manabí. Ecuador ha hecho frente a estos problemas a través de la preparación y capacitación de los docentes para contribuir con el afianciamiento de la creatividad en la enseñanza, cuya misión primordial es la generación de conocimiento de forma creativa, responsable, competente y comprometida con el proceso de enseñanza-aprendizaje, esto se puede hacer posible mediante la toma de conciencia sobre procesos creativos para poder desarrollar una enseñanza creativa.

Esta investigación toma como referencia inicial el trabajo realizado por (López Díaz, 2017) sobre las estrategias de enseñanza creativa, donde se pretende identificar los conceptos del rol del Docente creativo en la enseñanza de una segunda lengua a través de una educación a distancia, determinar si existe alguna relación entre estos conceptos y determinar si se desarrolla una enseñanza creativa e innovadora. Se definen los desafíos del docente en la actualidad, sus necesidades de renovación 
constante, para generar nuevas acciones en su función formativa.

En cuanto a lo que a creatividad se refiere, se prioriza el que manifiesta (Ramón Fernández et al., 2019): "Hablar de creatividad es hablar de potencial humano $y$ de transformación social, es hablar de modelos de sociedad que tengan iniciativa y capacidad para resolver los problemas, es, en definitiva, hablar de progreso". Página 473. En base a este concepto nace la pregunta, que es la creatividad, y por experiencias personales y vivenciales se puede hilar lo siguiente; la creatividad es sinónimo de innovación, de estudio permanente, de investigación, de experimentación, etc. con la finalidad de obtener resultados para impulsar el desarrollo del ser humano en las diferentes facetas donde le toca interactuar para dar solución a los diferentes planteamientos que surgen de acuerdo a cada interrogante que se presente en el universo de su vivir.

Considerando las aportaciones de (Maestro, 2019). Que nos dice: “Un ser es creativo cuando se encuentra incentivado, posee conocimientos para crear y cierta flexibilidad mental; en la educación formal se refuerza la tendencia hacia el pensamiento más estructurado, donde se utilizan técnicas de enseñanza creativas que motiven siempre a reflexionar $y$ conlleven a pensar de manera original, canalizando siempre hacia la resolución de problemas”. Página 8

Entonces la importancia del Rol del Docente creativo en el proceso educativo se convierte en el eje en el cual giran multiplicidad de actividades, conceptos, observaciones, experimentaciones, investigaciones, etc.

Para (Daniela et al., 2018) “El papel de los educadores, preparados, comprometidos $y$ dispuestos a utilizar diferentes tecnologías para aprendizaje, está en el centro del proceso educativo" página 3

Por ello la educación a distancia constituye una alternativa para enfrentar este enorme reto, ya que es considerada como una mediación pedagógica, capaz de promover y acompañar el aprendizaje de los que participan en este proceso de construir y apropiarse del conocimiento, convirtiéndose en una nueva perspectiva, cuyas funciones 
de desempeño recaen en las instituciones educativas, debido a la gran demanda existente por parte de la sociedad, sustentándose en el acogimiento de nuevas estrategias que permitan el desarrollo de la información, del conocimiento y del desarrollo cultural.

De acuerdo con Vygotsky (1932, 1995) citado por (FernándezCárdenas \& Silveyra-De La Garza, 2018), el aprendizaje no puede ser concebido como un producto individual, sino que es resultado de la participación social vinculada al uso de herramientas culturales. Como herramientas culturales pueden entenderse los sistemas de signos (lenguaje, escritura y sistemas de números) creados por las sociedades en el transcurso de la historia humana y el cambio de la forma de la sociedad, acorde con su nivel de desarrollo cultural.

Para ello es necesario apoyarse en lo que expone (Fernandez-Castillo, 2016) sobre las Estrategias de aprendizaje y adquisición de una segunda lengua, y estas parten desde un planteamiento derivado de la psicología cognitiva y meta cognitiva, considerando como estrategias primarias a aquellas que se establecen en base a un trabajo directo del sujeto con la información base de un aprendizaje, es decir serían aquellas que, sintéticamente, se relacionan con la recepción, memorización o almacenamiento y recuperación de información y las secundarias que consideran tipologías como las personales, socio-afectivas, ambientales, temporales así como la educación a distancia 0 virtual, estrategias primarias y estrategias secundarias. Se realizó una revisión bibliográfica relacionada con el rol docente, la educación.

La investigación hace referencia a los repositorios o archivos, de autores que han desarrollado investigaciones 0 estudios relacionados con el rol del docente creativo, la enseñanza de una segunda lengua, así como de la educación a distancia, la importancia y sus estrategias para el buen desarrollo del proceso educativo.

\section{Métodos}

La investigación tuvo un enfoque cuali - cuantitativo, debido a que se emprendió el tema de estudio desde los aspectos cuantitativos y confía en 
la medición numérica y desde los aspectos cualitativos, cuyo interés se centró en el estudio de los significados de las acciones humanas; con el fin de identificar manifestaciones de suma importancia que sirvieron de sustento a la investigación en cuanto al aprendizaje de una segunda lengua. Así también se utilizó la investigación bibliográfica, mediante el análisis de textos relacionados con el objeto de estudio, considerando los que se han realizado durante los últimos diez años, para obtener los contenidos más recientes de la información requerida.

Se implementó una investigación exploratoria que organizó las particularidades de orden descriptivo y analítico de las variables de estudio; valió para identificar manifestaciones que están relacionadas con el aprendizaje de una segunda lengua, poco investigados en el sector social, para ampliar el marco de conocimientos y llevar a efecto una investigación aplicada en lo relacionado con el rol del docente creativo en una educación a distancia. La población de la investigación correspondió a directivos, docentes y estudiantes de la U.E. "Francisco Pacheco" de la ciudad de Portoviejo. El tipo de muestra utilizado está constituida por conveniencia e informantes claves; tomando en cuenta, según lo señala (Otzen T. \& Manterola C., 2017) Técnicas de muestreo sobre una población a estudio. Permite seleccionar aquellos casos accesibles que acepten ser incluidos. Esto, fundamentado en la conveniente accesibilidad y proximidad de los sujetos para el investigador, facilitó la selección de personas por razones tales como: conocimiento del tema, por la disposición de compartir información, etc. Con esta definición se consideró a 3 directivos que conforman la parte administrativa de la Institución, 3 docentes del área de la segunda lengua y 40 estudiantes de Educación Básica Superior de la Institución. La investigación responde al método Analítico Sintético, el cual sirvió para seleccionar el objeto de estudio y analizó cada una de sus partes y sintetizar la información; Inductivo Deductivo porque sirvió para construir una investigación sólida y fundamentada adecuadamente a nivel teórico. 
La información primaria fue proporcionada por los directivos, docentes y estudiantes de la Institución, como fuente secundaria se contó con repositorios de Google académico de universidades, libros digitales, artículos científicos. Las técnicas de procesamiento y análisis de los resultados que se utilizaron fueron métodos estadísticos, mediante la recolección de datos por medio de cuestionarios de encuestas con preguntas de base estructuradas a estudiantes, así como las entrevistas a directivos y docentes, para evidenciar los resultados de la investigación.

\section{Resultados y discusión}

De acuerdo a los resultados obtenidos para identificar las competencias que debe asumir el docente creativo para la enseñanza de una segunda lengua se consideraron los aportes de las entrevistas y encuestas realizadas a directivos, docentes y estudiantes.

El $34,6 \%$ de los estudiantes respondieron que las estrategias que aplican los docentes para desarrollar las clases de la segunda lengua de manera virtual son creativas, estimulan el aprendizaje y motivan la participación.

El $33,3 \%$ de los estudiantes que participaron en la encuesta respondieron que los docentes para motivar el aprendizaje de una segunda lengua utilizan dinámicas motivacionales.

Tabla 1. ¿Cómo considera las estrategias que aplican los docentes para desarrollar las clases de la segunda lengua de manera virtual?

\begin{tabular}{clc}
\hline $\mathbf{N}^{\circ}$ & Alternativas & Porcentajes \\
\hline A & Anticuadas & $3,8 \%$ \\
B & Creativas & $34,6 \%$ \\
C & Motivan la participación & $29,5 \%$ \\
D & Estimulan al aprendizaje & $32,1 \%$ \\
\hline TOTAL & & $100 \%$ \\
\hline
\end{tabular}


Tabla 2. ¿Qué hacen los docentes para motivar el aprendizaje de una segunda lengua a través de la educación a distancia?

\begin{tabular}{clc}
\hline $\mathbf{N}^{\circ}$ & \multicolumn{1}{c}{ Alternativas } & Porcentajes \\
\hline A & Selecciona videos musicales o documentales & $30,8 \%$ \\
B & Programa sesiones de interacción en Skype & $7,7 \%$ \\
C & Utiliza dinámicas motivacionales & $33,3 \%$ \\
D & Maneja herramientas didácticas interactivas & $28,2 \%$ \\
\hline TOTAL & & $100 \%$ \\
\hline
\end{tabular}

Según González Hinojosa (2015) Las implicaciones de aprender una lengua extranjera son muchas y muy variadas. Desde la correcta gramática y pronunciación hasta contar con un amplio vocabulario; el estudiante debe desarrollar ciertas aptitudes con la ayuda de los docentes, imbuirse en un ambiente creativo, que estimulen el aprendizaje, para efectos de aplicar sus conocimientos y ser capaz de transmitir su mensaje en la lengua deseada.

Se espera que los docentes sepan con certeza que sus decisiones pedagógicas influyen en el desarrollo de las capacidades en sus estudiantes, por ello se deben empoderar y asumir el desafío permanente de hacerse cargo de sus prácticas de una manera proactiva y trabajando en equipo, asumiendo que solo los esfuerzos conjuntos lograrán el impacto esperado en los estudiantes.

En lo referente a distinguir las características que deben prevalecer en el rol docente para una educación a distancia, se deben considerar los conocimientos tecnológicos adquiridos para el desarrollo y aprendizaje fundamentales para el proceso educativo, ya que estas técnicas resultan agradables en el momento de la enseñanza aplicando ejercicios orales.

El $75,6 \%$ de los estudiantes supieron responder que los conocimientos tecnológicos adquiridos para el desarrollo y aprendizaje de una segunda lengua son fundamentales para el aprendizaje.

El $52,6 \%$ de los estudiantes encuestados respondieron que las técnicas que le agradarían que se 
utilizaran en el proceso de enseñanza de una segunda lengua serían los ejercicios orales.

Tabla 3. ¿Los conocimientos tecnológicos adquiridos por Ud. para el desarrollo y aprendizaje de una segunda lengua, son?

\begin{tabular}{clc}
\hline $\mathbf{N}^{\circ}$ & Alternativas & Porcentajes \\
\hline A & Fundamentales para el aprendizaje & $75,6 \%$ \\
B & Sustentos prácticos para el desempeño & $17,9 \%$ \\
C & Habilidad para optimizar acciones & $5,1 \%$ \\
D & Fuente de capacidad instrumental & $1,3 \%$ \\
\hline TOTAL & & $100 \%$ \\
\hline
\end{tabular}

Tabla 4. ¿Qué técnicas le agradaría que se utilizaran en el proceso de enseñanza de una segunda lengua?

\begin{tabular}{clc}
\hline № & Alternativas & Porcentajes \\
\hline A & Analogías y contrastes & $10,30 \%$ \\
B & Conexión de significados & $19,20 \%$ \\
C & Ejercicios orales & $52,60 \%$ \\
D & Enfoque cognitivo & $12,80 \%$ \\
E & Aprendizaje Kinestésico (juegos) & $5,10 \%$ \\
\hline TOTAL & & $100 \%$
\end{tabular}

Según María V. M. (2014) citado por (Burdano, 2018) en la enseñanza aprendizaje se deben practicar las técnicas que permitan desarrollar las capacidades comunicativas y facilitarle la posibilidad de interactuar mediante ellas. Todo recurso didáctico para la enseñanza aprendizaje tiene un objetivo pedagógico al ser diseñado y utilizado, porque cumple la función fundamental, de ayudar al docente a mediar los conocimientos y al estudiante la adquisición de habilidades en el dominio de la segunda lengua.

En el ámbito de los campos de formación académica requiere adquirir cada docente para tratar los contenidos que se desean aprender, su pedagogía y didáctica, acogerse a técnicas, que permitan desarrollar el pensamiento crítico y creativo, el aprender de manera autónoma, 
mostrando iniciativa para

autorregularse $y$ fortalecer su desarrollo personal, utilizando las tecnologías de la información y la comunicación de manera crítica y aplicando sus habilidades lingüísticas y comunicativas en diversos contextos.

$Y$ para definir los recursos metodológicos y didácticos que el docente debe utilizar para alcanzar los aprendizajes con sus estudiantes, se requiere utilizar como una de las técnicas más adecuadas, el método interactivo de capacitación a través de juegos (Kinestésico), así como el enfoque cognitivo por considerarlo indispensable en el desarrollo del conocimiento.

Según (Burdano, 2018) en la enseñanza aprendizaje se deben practicar las técnicas que permitan desarrollar las capacidades comunicativas y facilitarle la posibilidad de interactuar mediante ellas.

En la formación académica se requieren docentes que deseen aprender y profundizar su pedagogía, su didáctica, acogerse a técnicas, que permitan desarrollar el pensamiento crítico y creativo, el aprender de manera autónoma, mostrando iniciativa para autorregularse $y$ fortalecer su desarrollo personal, utilizando las tecnologías de la información y la comunicación de manera crítica y aplicando sus habilidades lingüísticas y comunicativas en diversos contextos.

\section{Conclusiones}

El Docente creativo ha asumido con responsabilidad, exactitud y fluidez la tarea de la enseñanza de una segunda lengua, aplicando las diferentes estrategias comunicativas que ha creído conveniente para los momentos de clase, apoyándose en una serie de habilidades y destrezas para desarrollar las competencias en los estudiantes, teniendo como fundamento principal, el uso de tecnologías de la información y comunicación, que le ha servido como herramienta mediadora en el proceso de enseñanza aprendizaje de una segunda lengua, sus conocimientos están en evolución, por ello ha considerado siempre la creatividad y la innovación para fortalecer su perfil profesional. 
Los docentes han definido los recursos metodológicos y didácticos recurriendo a interacciones tradicionales, que sirven de base para fortalecer así, la motivación y convirtiendo el ambiente de aprendizaje en un espacio que da cabida a: estrategias organizativas, enfocadas en la comunicación, cooperación, socialización e intercambio de experiencias; y las cognitivas dirigidas a apoyar los procesos de pensamiento como la activación de conocimientos previos, la creatividad, la organización de contenidos y la resolución de problemas, que, con el apoyo de recursos didácticos favorecen el ambiente educativo cuyo escenario interactivo, innovador, da lugar a que los momentos de clase sean participativos entre el docente y los estudiantes.

\section{Bibliografía}

Burdano, M. J. (2018). EL APRENDIZAJE COOPERATIVO Y LA INTERACCIÓN SOCIAL ENTRE PARES EN LOS ESTUDIANTES DE 8VO 9NO $Y \quad 10 M O$ AÑO DE EDUCACIÓN GENERAL BÁSICA DE LA UNIDAD EDUCATIVA PELILEO
PROVINCIA TUNGURAHUA CANTÓN

PELILEO [Universidad Técnica de Ambato].

https://repositorio.uta.edu.ec/j spui/bitstream/123456789/28

360/1/María José Burbano C.C. 1803207545.pdf

Daniela, L., Visvizi, A., GutiérrezBraojos, C., \& Lytras, M. D. (2018). Sustainable Higher Education and TechnologyEnhanced Learning (TEL). https://doi.org/10.3390/su101 13883

Fernández-Cárdenas, J. M., \& Silveyra-De La Garza, M. L. (2010). Disciplinary knowledge and gesturing in communicative events: A comparative study between lessons using interactive whiteboards and traditional whiteboards in mexican schools. Technology, Pedagogy and Education, 19(2), 173-193. https://doi.org/10.1080/14759 39X.2010.491219

Fernandez-Castillo, A. (2016). Estrategias de aprendizaje y adquisición de una segunda lengua. ReiDoCrea, 4, 391404.

González Hinojosa, A. R. (2015). Estrategias de enseñanza del idioma inglés utilizando la producción oral (Tesis de posgrado) [Tecnológico de Monterrey].

https://repositorio.tec.mx/bitst 
ream/handle/11285/626572/A Ima_Rosa_González_Hinojos a_.pdf?sequence $=1$

López Díaz, R. A. (2017). Estrategias de enseñanza creativa (Primera ed). Universidad de La Salle. http://biblioteca.clacso.edu.ar/ Colombia/fceunisalle/20180225093550/est rategiasen.pdf

Maestro, W. del. (2019). Cómo lograr ser un docente creativo en el aula.

https://webdelmaestrocmf.co $\mathrm{m} /$ portal/como-lograr-ser-undocente-creativo-en-el-aula/

Otzen T. \& Manterola C. (2017). Técnicas de muestreo sobre una población a estudio Buscar con Google. International Journal of Morphology, 35, 227. https://www.google.com/sear ch?q=otzen+t.+\%26+mantero la+c.+(2017).+técnicas+de+ muestreo+sobre+una+poblac ión+a+estudio\&rlz=1C1JZAP esUS952US952\&oq=OTZE $\mathrm{N} \% 2 \mathrm{C}+\mathrm{T} .+\% 26+\mathrm{MANTEROL}$ $\mathrm{A}+\mathrm{C} \&$ aqs $=$ chrome $.1 .69 \mathrm{i} 57 \mathrm{j0i}$ 512l2j0i22i30.18237j0j7\&sour ceid $=$ chrome $\&$ ie $=U T F-8$

Ramón Fernández, J., Da Vinci, L., Einstein, A., Alva Edison, T., Atanasoff, J., Amadeus Mozart, W., Dalí, S., \& Ford Steve, H. (2019). Creatividad: Revisión del concepto Fátima Llamas Salguero-Universidad de Extremadura Mónica
Gutiérrez Ortega-Universidad Internacional de la Rioja. REIDOCREA, 8, 467-483. https://reunir.unir.net/bitstrea m/handle/123456789/9783/Ar ticulo_creatividad.pdf?sequen ce $=1$ \&isAllowed $=y$ 